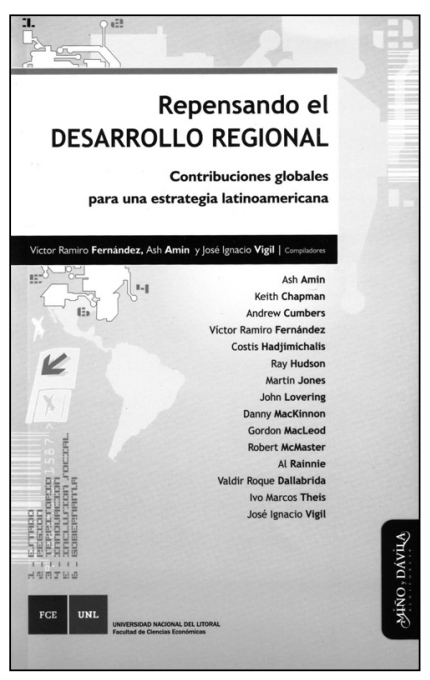

Victor Ramiro Fernández, Ash Amin y José Ignacio Vigil (Comps.)

\section{Repensando el desarrollo regional. Contribuciones globales para una estrate- gia latinoamericana}

Buenos Aires: Miño \& Dávila (2008)
Es un hecho indiscutible que en las últimas dos décadas los aspectos atinentes al territorio, a la localización espacial de las actividades económicas y a las dinámicas sociales de orden subnacional, se han convertido en referentes fundamentales de los procesos sociopolíticos, tanto al interior de los países como a escala global.

Es aparentemente una paradoja, que justo cuando la globalización sugería el "fin de la geografía", en el sentido de que los límites político-administrativos de los entes estatales se estarían banalizando, se haya producido simultáneamente una revalorización de la dimensión territorial, no sólo en el plano teórico sino en el de la práctica de las políticas públicas y las estrategias empresariales.

La cuestión es que en la medida en que los profundos procesos de cambio en los planos tecnológico, económico, cultural y político a escala mundial impactan inevitablemente en los territorios subnacionales, los dos procesos -globalización y localización- se convierten en las dos caras de una misma moneda. Tanto es así que la valorización de lo local en relación dialéctica con lo global ha dado lugar a neologismos como "glocal" y "fragmegración", para tratar de expresar la pertenencia de los dos ámbitos espaciales al mismo campo relacional: la globalización de lo local y la localización de lo global.

A la re-espacialización del acontecer socioeconómico y político y sus caleidoscópicas implicaciones teóricas e institucionales, está dedicado el libro colectivo intitulado "Repensando el desarrollo regional. Contribuciones globales para una estrategia latinoamericana”. Compilado por Víctor Ramiro Fernández, Ash Amin y José Ignacio Vigil, ellos mismos autores de varios de los capítulos que lo integran, este denso y abigarrado volumen, constituye un aporte de la más alta calidad y pertinencia al análisis de una temática que está deviniendo una condición esencial de viabilidad para el desarrollo futuro de América Latina.

Así lo están demostrando claramente en el ámbito andino, por ejemplo, los recientes y accidentados procesos constituyentes en Bolivia y Ecuador, en los cuales la cuestión territorial fue uno de los escollos más difíciles de superar. 
La calidad de la obra deriva no sólo del contenido intrínseco de los 18 capítulos que lo integran, sino del riguroso plan metodológico global en el que se fundamenta. A diferencia de lo que suele ocurrir con colectivos de esta extensión (557 páginas), que anteponen la cobertura a la coherencia, este trabajo está montado sobre una rigurosa estructura general, que le confiere a todo su contenido un sentido de unidad y articulación orgánica.

Dicho diseño metodológico se despliega secuencialmente en tres planos: a) la presentación objetiva y "neutral" (hasta donde ello es posible) de lo que los compiladores llaman la "Nueva Ortodoxia Regionalista”-NOR-, b) una discusión crítica sobre los alcances y limitaciones de la NOR, y c) el planteamiento de una visión alternativa relevante para América Latina. El empeño por la coherencia global de la obra se pone especialmente de manifiesto en los capítulos de contexto ( 1 y 2 ), en el dedicado a la plataforma para pensar alternativamente las especificidades de América Latina (9) y en el de Conclusiones, en los que los compiladores hacen un esmerado esfuerzo para "entramar" y correlacionar los diversos artículos del libro, a través de abundantes referencias cruzadas entre ellos.

Frente a la reiterada queja contra los modelos teóricos importados y la ausencia de pensamiento propio que abunda en nuestro medio, pero que generalmente no desemboca en el planteamiento de propuestas alternativas, el descrito esquema metodológico debería ser de más frecuente utilización.

Adentrándonos en el texto en mención, las tres preguntas que permean su contenido desde el principio introductorio hasta las conclusiones son las siguientes:

¿Cómo explicar la paradoja que se configura con la revalorización (rejerarquización) del discurso teórico de la dimensión regional-local del desarrollo y la simultánea agudización de los desequilibrios territoriales? ¿Cuáles son las deficiencias presentes en la nueva ortodoxia regionalista? ¿Cuáles serían los elementos de una alternativa teórica y de políticas públicas relevante para América Latina?

En el intento de responder a dichas cuestiones centrales, el libro dedica a cada una de ellas una parte (para un total de tres), las cuales a su vez están integradas por un número variable de capítulos.

En cuanto a lo primero -la acuciante paradoja del reposicionamiento del discurso teórico regionalista- ensanchamiento de las asimetrías interterritoriales- en el capítulo 1, de la autoría de V. R. Fernández, A. Amin y J. I. Vigil, se informa en una bien lograda síntesis sobre las diversas corrientes teóricas que confluyen en la NOR y en los capítulos 2, 3, 4, $5,6,7$ y 8 se discuten en profundidad los límites, posibilidades y contradicciones de dicha nueva ortodoxia dominante. La argumentación es expuesta en forma que resulta convincente y persuasiva, sobre todo cuando se señala explícitamente que la NOR ha permeado la esfera de las instituciones públicas y ha producido un estamento de tomadores de decisiones y operadores que ofician acríticamente en el altar del nuevo fundamentalismo. No obstante, a la tesis de que los problemas de la realidad, en este caso la persistencia de los desequilibrios territoriales, se deben a fallas en la teoría ("las inconsistencias del concepto 
teórico constituido en la nueva ortodoxia”, p.14), habría que ponerle una nota de cautela. Ello equivale a que Marx hubiera atribuido las contradicciones del capitalismo que analizó en el siglo XIX, a fallas en las teorías de los economistas clásicos de la época o que se sostenga que la crisis financiera actual tenga su causa principal en las teorías neoclásicas contemporáneas. Por supuesto, que las teorías tienen a veces un impacto significativo en los acontecimientos reales, sobre todo si, como en el caso que nos ocupa, ellas devienen en un paradigma compartido por las élites dominantes, los gobiernos, las instituciones supranacionales y los agentes económicos. Pero, en un fenómeno como el de las desigualdades inter-territoriales intervienen otros factores causales, entre los cuales el más determinante ha sido, sin duda, la propia globalización, que ha favorecido principalmente a las regiones que de entrada tenían una mejor plataforma competitiva. Así, si bien es indiscutible que en varios países latinoamericanos la descentralización fiscal puesta en práctica en los últimos dos decenios, ha mejorado el acervo de capital humano (salud y educación) de las regiones, ello no ha sido suficiente para compensar las nuevas inversiones en servicios avanzados y otros sectores transables en las grandes áreas metropolitanas.

Por otra parte, en lo que hace al problema de las brechas, la nueva geografía económica a la Krugman, por ejemplo, proporciona un marco teórico basado en mecanismos de causación circular acumulativa, que permiten diagnosticar y medir adecuadamente el problema. Otra cosa es que no se actúe en consecuencia.

Por lo demás, la tendencia hacia la autonomización de las regiones (el "enclaustramiento territorial"), a la que se hace referencia insistentemente en el libro, no es simplemente un artefacto ideológico de la NOR, sino una de las facetas del complejo proceso de reescalamiento que está experimentando el Estado y que se encuentra, por cierto, muy bien tratada en varios artículos del libro (MacLeod; Jones; Amin; McKinnon; Cumbers y Chapman; y Theis) y explícitamente recogido en el capítulo de conclusiones. En este sentido el llamado de Mcleod (cap. 3) y de Theis (cap. 9) a incorporar las perspectivas analíticas de la escuela de la regulación francesa al debate sobre la NOR, parece muy pertinente. Según estos enfoques, compartidos en la academia anglosajona por Jessop y Wolfe, entre otros, así como el Estado Keynesiano de Bienestar-EKB- era la forma de regulación más apropiada para la reproducción ampliada del fordismo, el Estado Shumpeteriano de Trabajo -EST - proporciona el mejor armazón político posible para el posfordismo. Ahora bien, siendo las funciones del ETB la promoción de la competitividad, la innovación y la flexibilización del mercado laboral, que son procesos cada vez más localizados, los espacios regionales resultan ser los escenarios más funcionales para el despliegue de sus actividades.

En otro orden de ideas, la fragmentación de antiguos Estados nacionales ya no es sólo una tendencia sino un hecho cumplido en ámbitos como la ex Unión Soviética, Checoslovaquia y Yugoslavia, como bien lo ha hecho notar Kenichi Ohmae.

En lo que concierne a las deficiencias teóricas de la NOR, que es, como se dijo, el segundo eje temático central del libro, éste comienza (en su capítulo 1) por hacer un prolijo survey de las corrientes que a juicio de sus autores (Fernández, Amin y Vigil) son los principales afluentes teóricos de la NOR: el enfoque de la acumulación flexible, el de los clusters a la 
Porter y diagonalmente, el de la Nueva Geografía Económica. La fusión de estos tres vectores (especialmente de los dos primeros) deriva, según Fernández, Amin y Vigil, en una "plataforma común" sustentada en "dar prioridad, primero analítica y luego política, al volumen y calidad de las articulaciones cooperativas y en general a las relaciones no mediadas por las relaciones de mercado (redes) entre los actores empresariales, gremiales, gobiernos locales, ONG, instituciones científicas, etc.”.

Además de las tres corrientes identificadas y analizadas por los autores en mención, cabría pensar en otros dos afluentes de la NOR. Por un lado, la perspectiva ambientalista, cuya preocupación por la conservación y uso adecuado del capital natural, se ha plasmado en el ubicuo concepto de "desarrollo sostenible"; y por otro, la fuerte tendencia hacia la descentralización política que ha estado al alza en América Latina en los últimos decenios.

En cuanto a lo primero, en la medida en que los recursos naturales están por definición localizados, la sostenibilidad del desarrollo tiene ineludiblemente una clara connotación territorial (en la contribución de Rainnie al libro, consignada en el capítulo 7, se menciona la pertinencia de incluir la dimensión ambiental en la NOR). Surge aquí el enfoque biorregional como una manera de asegurar los equilibrios ecológicos y el adecuado aprovechamiento del capital natural tanto a escala regional, como nacional y transnacional. Con respecto a la descentralización en sus diversas acepciones, que se ha elevado recientemente al rango constitucional en la mayoría de los países andinos, por ejemplo, se trata de una tendencia que ha sido recurrente en América Latina desde los albores de la vida republicana y que a pesar de hundir sus raíces en condicionantes históricos que trascienden la moda de la nueva ortodoxia regionalista, viene a reforzarla.

Presentada en el capítulo 1, la sinopsis teórica de la NOR y una completa panorámica de la forma en que ella está permeando las concepciones y prácticas de los organismos internacionales, con una referencia especial a lo que ocurre en América Latina, el libro trae un capítulo 2 (Fernández, Amin y Vigil) que constituye una síntesis afortunada del contenido de todo el resto del volumen. La lectura de este capítulo permite identificar los tres ejes principales del debate y las propuestas que se avecinan: el grado de novedad de los aportes teóricos de la NOR; sus riesgos y debilidades; y los elementos de una plataforma alternativa.

\section{Los aportes teóricos novedosos.}

Sobre este tema, emerge de los diversos artículos un cierto consenso alrededor de las tesis de Amin, según las cuales "el Nuevo Regionalismo ofrece una solución basada en la movilización de los recursos locales... un planteo que abarca desde una nueva política industrial y... proyectos de consolidación de las economías locales de asociación hasta la adopción de medidas encaminadas a mejorar el poder de reflexión institucional, el potencial de aprendizaje y la creatividad local". Aunque con diferentes matices y cualificaciones de diverso grado, McLeod (cap. 3), Hadjimichalis (cap. 5) y Rainnie (cap. 7) están básicamente de acuerdo con Amin en que la NOR ha aportado una rica gama de conceptos y elementos analíticos para pensar las regiones en el marco de la globalización, distintos tanto de la ortodoxia neoclásica como del centralismo planificador de los años '60 y '70. La voz radicalmente 
disidente aquí es la de Lovering (cap. 3), quien, basado en sus experiencias en Gales, sostiene que la NOR no es "una alternativa radical al mainstream, sino que es el mainstream" y que su influencia en el desarrollo regional puede reducir en vez de ampliar sus horizontes intelectuales y estratégicos.

\section{Riesgos y debilidades.}

Desconocimiento de los condicionantes históricos (path dependences) en los que se inscribe el desarrollo regional (Amin; Mckinnon, Cumbers y Chapman; y Fernández); "reificación" de la dimensión regional que conduce a un hiperlocalismo que ignora el contexto macroeconómico e internacional (Amin, McLeod; McKinnon, Cumbers y Chapman, Cumbers, McKinnon y McMaster; y Hudson) y legitimación del desmantelamiento de las pautas redistributivas que favorecían el trabajo y las regiones rezagadas, propias del Estado Keynesiano de Bienestar (McLeod), son algunas de las críticas más incisivas que se formulan contra la NOR en el libro. Al lado de estas también cabría mencionar las de Hadjimichalis (cap. 5), quien resiente la utilización en la NOR del concepto de "capital social", en una acepción ingenua y vacía de sus connotaciones políticas conflictivas (siguiendo a R. Putnam) y en general, el tratamiento despolitizado de los factores no económicos (sociales y culturales) de lo regional, que ignora su inserción en unas estructuras de poder asimétricas e inequitativas.

Argumentos similares a los de Hadjimichalis, esgrimen Hudson (cap. 6) y McKinnon, Cumbers y Chapman (cap. 6), pero en estos casos a propósito de la incorporación a la NOR de los conceptos de innovación, conocimiento y aprendizaje.

Llama la atención que siendo el tema de la agudización de los desequilibrios territoriales, uno de los términos de la paradoja que constituye el leitmotiv del libro, esta cuestión no está presente explícitamente en ninguno de los artículos del capítulo 3, dedicado a la discusión crítica de la NOR. El registro sobre este asunto es especialmente fuerte en las contribuciones escritas a trío por los compiladores de la obra (Fernández, Amin y Vigil).

Por su parte, Fernández (cap. 9) también introduce una acerba crítica a la forma en que la Unión Europea ha incorporado la NOR en sus políticas regionales, que puede resultar un tanto desbalanceado. Si bien es cierto, como lo señala nuestro autor, que, en general, el proyecto global de integración europeo y en particular, el de cohesión regional, fueron colonizados respectivamente por la lógica neoliberal y por la NOR, no lo es menos que en el esquema europeo existe, por un lado, una política activa de redistribución a favor de las regiones rezagadas y por otro, un modelo de gobernanza que responde claramente a los principios de multiescalaridad que con razón se reclaman a lo largo y ancho de la obra comentada.

Lo primero responde a una preocupación permanente, que se remonta al propio Tratado de Roma de 1958, por la reducción de las diferencias entre los niveles de desarrollo de las diversas regiones y el apoyo a las más atrasadas, que se concretó en la existencia de unos Fondos Estructurales y de Cohesión creados en la década de los 70 y que se mantienen hoy plenamente operativos. En la pretendida “difusión del poder del Estado nacional” en el sistema de toma de decisiones europeo, lo que en realidad existe en este contexto es un complejo entramado de 
niveles de decisión, donde los Estados miembros conservan su capacidad decisoria en múltiples asuntos claves, como la Política Exterior y de Seguridad Común y el manejo mismo de los Fondos Estructurales. En este proceso el margen de maniobra de los órganos supranacionales es muy limitado y rigen estrictamente los principios de subsidiariedad y complementariedad, según los cuales no puede hacerse en un determinado nivel lo que sea posible realizar eficazmente en un nivel inferior.

Con la emergencia del proyecto de "Europa de los regiones" y la creación como órgano consultivo al más alto nivel del Comité de la regiones (1994), el sistema de toma de decisiones europeo puede describirse con el concepto de multi-level governance ${ }^{1}$, cuya esencia es una de las falencias que se le anotan a la NOR y cuya conveniencia en una plataforma alternativa es una de las propuestas que con mayor insistencia se plantea. Más aún, la política regional europea ha ido evolucionando hacia una concepción mas integral en el marco de una "Perspectiva Europea de Desarrollo Espacial" (ESDP)², que al lado de la competitividad y la conservación de los recursos naturales mantiene la Cohesión Social como uno de sus pilares fundamentales. A su vez y desde el punto de vista conceptual y teórico, la ESDP ha dado lugar a la búsqueda académica de unas Spatially Integrated Social Sciences, que constituyen una buena base para la teoría holística e integradora que proponen algunos de los autores del libro (McLeod, cap. 3).

\section{Propuestas para una plataforma alternativa}

Tramitado el debate sobre las fallas y limitaciones de la NOR, el libro pasa en sus capítulos 8 y 9 a proponer insumos para superar tales deficiencias y avanzar hacia una plataforma alternativa, con relevancia para América Latina. En esencia, las propuestas son del siguiente tenor:

- La recuperación del "sentido de la economía politica", mediante el reconocimiento de que los procesos regionales no están insertos en un escenario utópico en donde predomina la armonía, la confianza y la reciprocidad, sino en un campo de fuerzas estructural y dinámicamente contradictorio y asimétrico, que condiciona desigualmente las posibilidades de las regiones (Theis, cap. 9). En los capítulos 5, 6, 7, 8 y 9, sus autores celebran la incorporación en la NOR de factores como las instituciones, el capital social, la innovación, el aprendizaje y las redes, pero abogan vehementemente por su inserción —en la vena de la Escuela de la Regulación Francesa y de Bourdieu- en un contexto que no ignore las distintas formas de lucha por el poder que afectan las regiones.

- La introducción de una "perspectiva relacional", en la que lo regional-local deja de ser percibido como un ámbito predefinido y autosuficiente con vínculos unidireccionales con su entorno, para pasar a entenderse como el resultado (la condensación) de un entramado

\footnotetext{
1 O sea una "política regional multipolar" con "instancias multilocalizadas" en los términos que sugiere Amin (cap. 8, segundo artículo).

2 La sigla en inglés de European Spatial Development Perspective.
} 
de relaciones multiescalares e interdependientes (flujos y redes). Es decir en esta visión el espacio no es un stock sino un flujo (Amin, cap. 8, Fernández y Vigil, cap. 9 y Fernández y Dallabrida, cap. 9).

- El acoplamiento a la mencionada "perspectiva relacional", de una "política regional multipolar" que opere tanto desde las regiones como fuera de ellas, a través de instancias de decisión multilocalizadas. Se impone concebir las regiones y localidades como entes "insertos en sistemas globales de producción, intercambio y conocimiento", que persiguen su avance y mejoramiento a través de múltiples formas de relacionamiento que van más allá de lo local.

- La reactivación del papel del Estado nacional. En el marco de la "perspectiva relacional" y de "la política regional multipolar", recuperar el papel del Estado nacional que resulta indispensable para los propósitos de una mayor justicia distributiva entre regiones e individuos (Fernández y Vigil, cap. 9 y Fernández y Dallabrida, cap. 9).

- La convergencia de los conceptos de "economía política", "perspectiva relacional", "politica regional multipolar" y "reactivación del Estado nacional" en el constructo "Sistema Social de Producción", que dé cuenta de una particular configuración regional en el contexto de una relación "macro-meso más realista e integradora, recuperando el papel del Estado nacional y las trayectorias de modelos nacionales, así como proyectos de cobertura sociales universales y acuerdos colectivos de concertación entre capital y trabajo" (Fernández y Vigil, cap. 9 y Fernández y Dallabrida, cap. 9).

La pertinencia de las propuestas que acaban de relacionarse es indiscutible, no obstante no sobra insistir en que el modelo de la política regional europea sí puede proporcionar algunos elementos válidos de referencia para la "política regional multiescalar" que se plantea. En el propósito del aggiornamento de la NOR cabría insinuar, además, la perspectiva de las "políticas secto-territoriales” elaborada por Pierre Müller, que trata precisamente de mostrar cómo las transformaciones del Estado han conducido a la adopción de una serie de políticas e instrumentos que participan híbridamente de las lógicas sectorial-vertical y espacial-horizontal, las cuales ya son de uso común en América Latina.

Con respecto a la búsqueda de una "nueva plataforma común” que sea "holística", "abarcativa", "integrada”, "relacional”, "multiescalar" y además verificada con exhaustivas investigaciones empíricas, es decir la "teoría final”, en la que, siguiendo a S. Weinberg, "todas las preguntas fundamentales hallarían respuesta sin requerir una explicación en términos de otros principios más profundos", ello resulta un tanto desmesurado, sobre todo en un campo como las políticas públicas regionales que requieren por fuerza de una buena dosis de ingeniería social.

Ya para terminar esta excesivamente larga reseña, sólo queda reiterar que el volumen en comento constituye un aporte de la más alta calidad y factura, que con toda seguridad será de gran valía para todos los interesados en el desarrollo regional de América Latina. La erudición, la amplitud de los objetivos, la capacidad de síntesis de sus compiladores y la bien lograda disposición de 
los artículos para lograr un texto orgánico, hacen de este volumen una referencia obligada en un campo cuya frondosa literatura comienza a ser difícil de manejar.

Edgard Moncayo Jiménez

\footnotetext{
* Economista, U. del Rosario, Bogotá, Colombia. Maestrías de las universidades de Harvard y Georgetown. Doctorado (c) de la Universidad Nacional de Colombia. Miembro de Número de la Academia Colombiana de Ciencias Económicas. Director del Centro de Investigaciones y Estudios de la Facultad de Ciencias Económicas, Administrativas y Contables de la Universidad Central, Bogotá D. C. Autor de una decena de libros y numerosos artículos sobre temas relacionados con el desarrollo económico, la integración latinoamericana y el desarrollo regional de Colombia y los países andinos. Ha ocupado altos cargos en el gobierno colombiano y en organizaciones internacionales, como la Comunidad Andina y la CEPAL.
}

Correspondencia: Edgard Moncayo Jiménez, calle 8, № 37-10, ap. 802, Edificio Lutaima, Barrio Corazón de Jesús, Bogotá D. C. E-mail: edgardmoncayo@yahoo.com. 